\title{
An Automatic High Efficient Method for Dish Concentrator Alignment
}

\author{
Yong Wang, ${ }^{1}$ Song Li, ${ }^{1}$ Jinshan Xu, ${ }^{1}$ Yijiang Wang, ${ }^{1}$ Xu Cheng, ${ }^{1}$ Changgui Gu, \\ Shengyong Chen, ${ }^{1}$ and Bin $\mathrm{Wan}^{3}$ \\ ${ }^{1}$ College of Computer Science, Zhejiang University of Technology, Hangzhou 310014, China \\ ${ }^{2}$ Department of Molecular Cell Biology, Laboratory for Neurophysiology, Leiden University Medical Center, 2300 RC, The Netherlands \\ ${ }^{3}$ Solarversal Technology, Hangzhou 310014, China \\ Correspondence should be addressed to Jinshan Xu; jxu@zjut.edu.cn
}

Received 13 February 2014; Revised 16 June 2014; Accepted 17 June 2014; Published 24 July 2014

Academic Editor: Carlo Cosentino

Copyright (C) 2014 Yong Wang et al. This is an open access article distributed under the Creative Commons Attribution License, which permits unrestricted use, distribution, and reproduction in any medium, provided the original work is properly cited.

\begin{abstract}
Alignment of dish concentrator is a key factor to the performance of solar energy system. We propose a new method for the alignment of faceted solar dish concentrator. The isosceles triangle configuration of facet's footholds determines a fixed relation between light spot displacements and foothold movements, which allows an automatic determination of the amount of adjustments. Tests on a $25 \mathrm{~kW}$ Stirling Energy System dish concentrator verify the feasibility, accuracy, and efficiency of our method.
\end{abstract}

\section{Introduction}

The constraint of limited fossil fuel resources and related environmental concerns have prompted the development of renewable clean energy. The solar energy, as one of the most promising options, has attracted emphasis from both industrial sections and theoretical studies $[1,2]$. The whole industry can be categorized into two genres: photovoltaic and thermal. The former takes the advantage that the photovoltaic materials can convert sun radiation into direct current electricity [3], while the latter uses simply the thermal energy of the sunlight. Concerning the relatively low energy flux on earth $\left(1000 \mathrm{~W} / \mathrm{m}^{2}\right)$, industrial applications typically utilize large surface areas of parabolic mirrors for concentrating energy to a receiver. For instance, the concentrator of the California Edison $25 \mathrm{~kW}$ dish/Stirling System utilizes spherically curve glass mirrors with a total surface area up to $90 \mathrm{~m}^{2}$, and reflection rate as high as $90 \%$ [4]. Such huge spherical mirrors are hard to be fabricated as a single unit. In practice, small facets are produced and assembled to form parabola shape, with each facet having approximately the same focusing point. To ensure an even distribution of energy flux to receiver, a proper alignment of facets is of crucial importance to the performance of a dish system [5].
Developing accurate and efficient alignment tools has been a hot research topic in the area of solar energy. Blackmon and Stone proposed a Digital Radiometer Alignment Method which contains a panel of computer controlled light sources and utilizes computer to interpret the reflected images [6]. However, it takes several hours or more to collect the image data, so as may not be efficient to accomplish real-time adjustment. In 2003, Andraka et al. at Sandia National Laboratory Albuquerque NM successfully implemented a color look-back approach to the dish alignment in a laboratory environment [7]. For field application, this method is a lit-tle time-consuming, typically taking 4-6 hours per dish. More recently, an alignment tool based on fringe reflection method has been applied to dish concentrator [8]. This automated alignment process requires preestablishment of a dish coordinate system, with the origin being the vertex of the parabola and $z$-axis along the optical line of the parabola. While in field application, the precise determination of optical line is impossible. As a consequence, installation errors are inevitable.

With the limitations of the previous approaches in mind, in this paper, we propose an automatic alignment method that allows a quick and accurate adjustment of facets. Although our discussion will be based on parabola dishes 


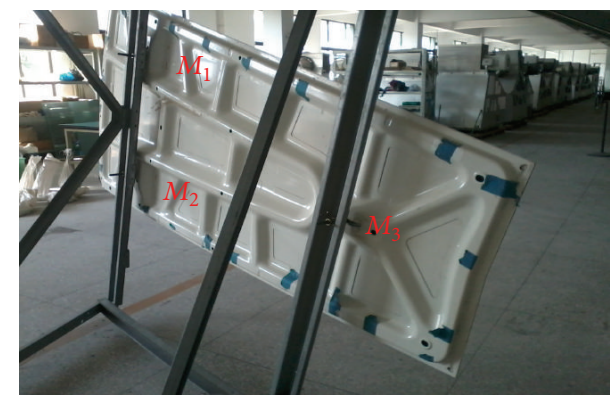

(a)

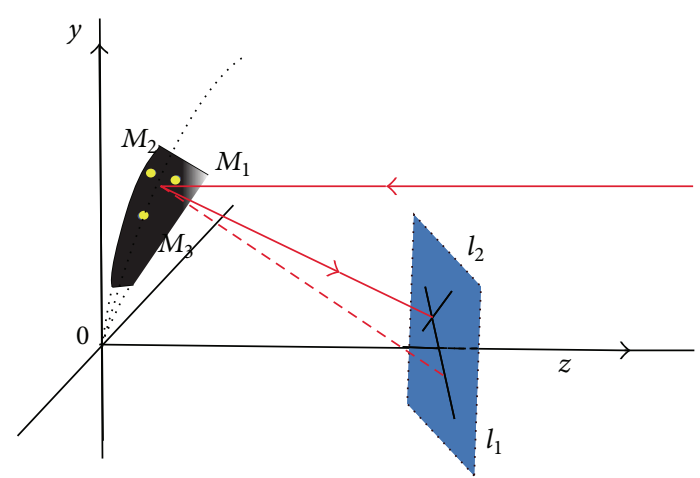

(b)

FIGURE 1: (a) Support structure of facets. Each facet is mounted with supports from three footholds $\left(M_{1}, M_{2}\right.$, and $\left.M_{3}\right)$, forming an isosceles triangle configuration. All of them can move along the surface normal at that point. The inset shows a zoom of the rectangular region labeled by the green dash lines. (b) Schematic illustration of the relation of the movements of footholds $M_{1}, M_{2}$ and the displacements of light spots on the screen. Moving the two bottom footholds $M_{1}, M_{2}$ in same direction; light spot moves along $l_{1}$ and along $l_{2}$ while moving in opposite direction.

with each facet supported by three footholds, the proposed method can easily be extended to dishes with other configurations. To better present the method, the rest of the paper is organized as follow: in Section 2, we present the mathematical bases of the method. Section 3 details the alignment procedure and presents some testing results. A conclusion and a discussion of the method will be given in Section 4 .

\section{Mathematical Bases}

Each facet is mounted with supports from three footholds, labeled as $M_{1}, M_{2}$, and $M_{3}$, as shown in Figure 1. The alignment of the facet can be achieved by adjusting the position of only two footholds, namely, $M_{1}$ and $M_{2}$, which can move along the direction of surface normals of the points. Noticing that the three footholds are aligned in isosceles triangle configuration, moving $M_{1}$ and $M_{2}$ simultaneously along the same direction, the facet rotates with $M_{3}$. As a consequence, the light spot of the reflected parallel light incident to the facet on a screen located near the focus would form a linear trajectory, $l_{1}$, as shown in Figure 1(b). While simultaneously moving $M_{1}$ and $M_{2}$ along opposite direction, the facet rotates along the bisector of $M_{1}$ and $M_{2}$ that passes $M_{3}$ (dashed line), resulting in the light spot moving along line $l_{2}$, which is almost perpendicular to $l_{1}$.

With this intuitive analysis, a strategy of moving a light spot $R\left(x_{R}, y_{R}\right)$ to a target position $T\left(x_{T}, y_{T}\right)$ can be proposed (see Figure 2): light spot $R$ is first moved to point $O$ along a line parallel to $l_{2}$; it is then moved to $T$ along a line parallel to $l_{1}$. Here the point $O\left(x_{0}, y_{o}\right)$ is the intersection of the two lines with

$$
\begin{gathered}
x_{o}=\frac{y_{T}-y_{R}+k_{2} x_{R}-k_{1} x_{T}}{k_{2}-k_{1}}, \\
y_{o}=\frac{k_{2} y_{T}-k_{1} y_{R}+k_{1} k_{2}\left(x_{R}-x_{T}\right)}{k_{2}-k_{1}},
\end{gathered}
$$

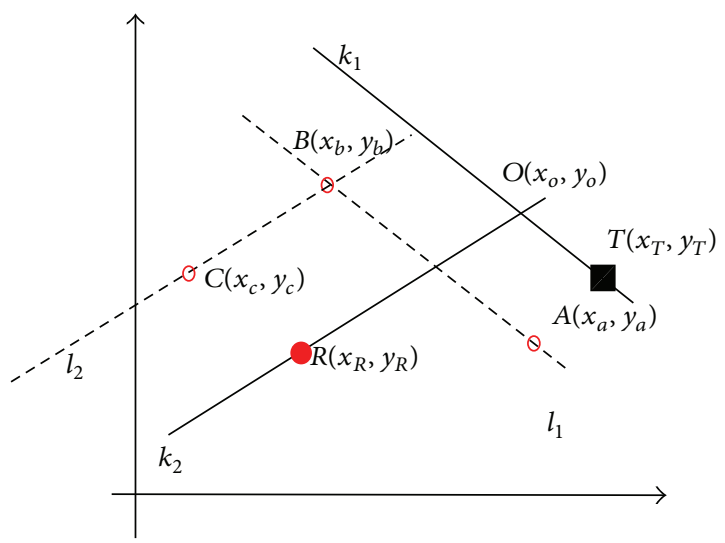

FIGURE 2: Schematic illustration of control strategy. A line passing through the target point $T$ and parallel to $l_{1}$ intersects the line that passes through current position $R$ with a slope the same as $l_{1}$ at point $O\left(x_{o}, y_{o}\right)$. The light spot reaches the target $T$ via the intermediate point $O . k_{1}, k_{2}$ are the slope of lines $l_{1}$ and $l_{2}$, respectively.

where $k_{1}$ and $k_{2}$ are slopes of lines $l_{1}$ and $l_{2}$, respectively. The motors' displacements that lead the light spot passing from $R$ to $O$ and eventually to $T$ are given by the following:

$$
\begin{gathered}
d_{M_{1}}^{R O}=\frac{\overrightarrow{R O} \cdot \hat{i}}{R_{l_{2}}}, \\
d_{M_{2}}^{R O}=-\frac{\overrightarrow{R O} \cdot \hat{i}}{R_{l_{2}}}, \\
d_{M_{1}}^{T O}=d_{M_{2}}^{T O}=\frac{\overrightarrow{O T} \cdot \hat{j}}{R_{l_{1}}} .
\end{gathered}
$$

Here unit vector $\hat{i}$ shows the direction of light spot when simultaneously moving $M_{1}$ forward (positive "+") and $M_{2}$ backward (negative "-") and $\hat{j}$ the direction of light spot 


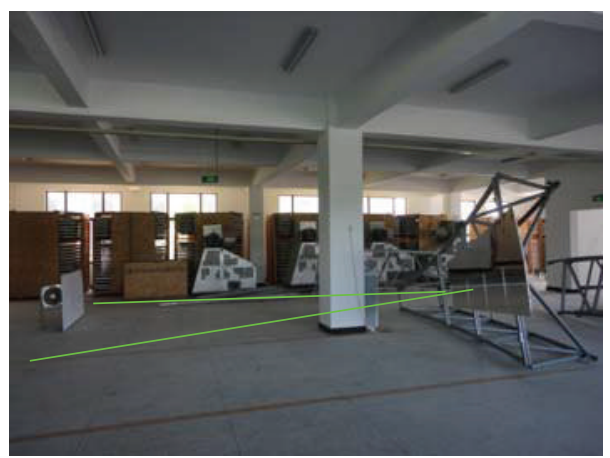

(a)

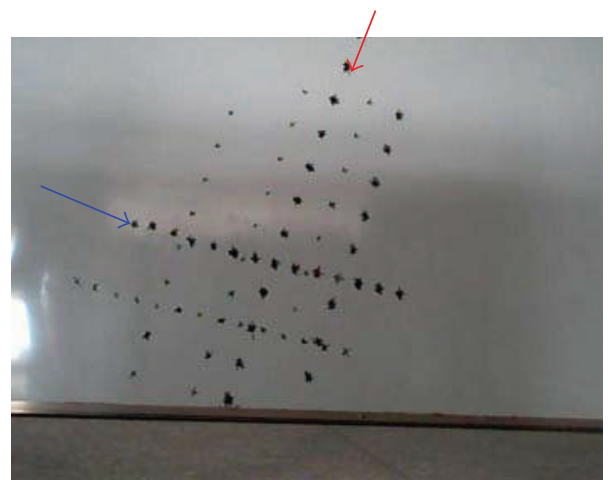

(b)

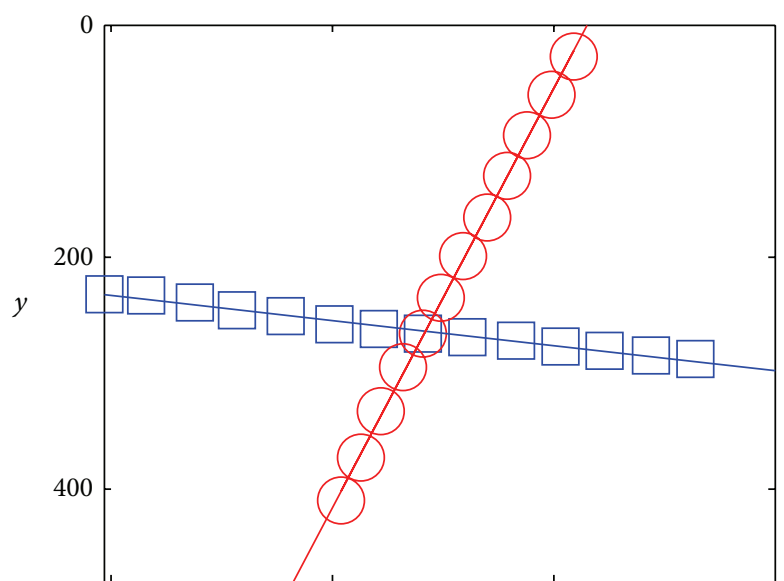

(c)

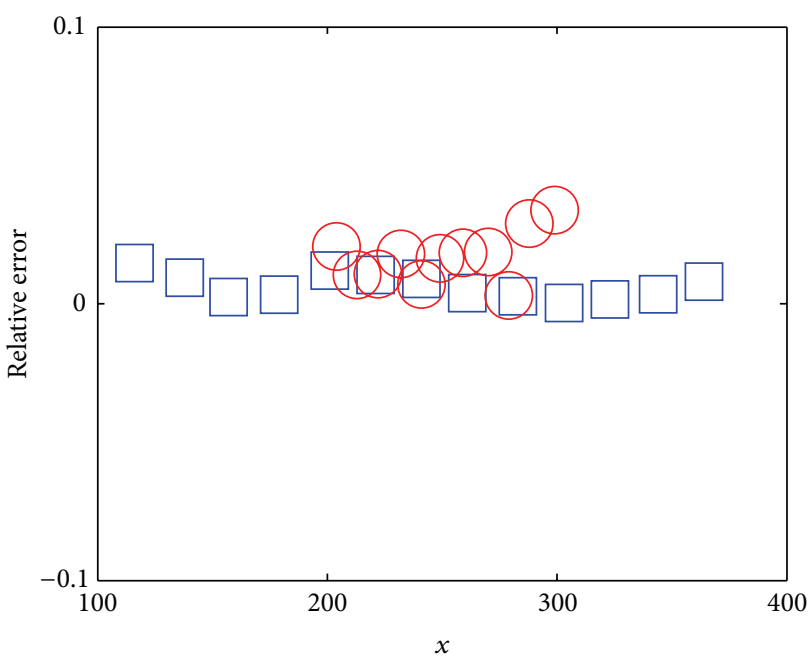

(d)

FIGURE 3: (a) Testing experiment setup. Light from a laser pointer is projected to the facets; its reflection is viewed on a screen near the focus. (b) Recorded positions of light spots by moving $M_{1}, M_{2}$ in same direction with interval of $2 \mathrm{~cm}$ (blue arrow) and in opposite direction with interval of $1 \mathrm{~cm}$ (red arrow). (c) The lights spots formed by moving $M_{1}, M_{2}$ can be well fitted by straight lines. Only the points indicated by arrows are plotted. (d) The relative errors to the fitted lines are of order $10^{-3}$.

when moving $M_{1}$ and $M_{2}$ both positively. For this reason, there is a minus sign of $M_{2}$ 's displacement $d_{M_{2}}^{R O}$. Based upon these analyses, the deviation of the light spot to the target can be eliminated by moving $M_{1}$ and $M_{2}$ with

$$
\begin{aligned}
& d_{M_{1}}=\frac{\overrightarrow{O T} \cdot \hat{j}}{R_{l_{1}}}+\frac{\overrightarrow{R O} \cdot \hat{i}}{R_{l_{2}}}, \\
& d_{M_{2}}=\frac{\overrightarrow{O T} \cdot \hat{j}}{R_{l_{1}}}-\frac{\overrightarrow{R O} \cdot \hat{i}}{R_{l_{2}}} .
\end{aligned}
$$

One may notice that (3) is derived based on a set of hypotheses: (1) moving motors simultaneously along the same direction/opposite direction at any initial states results in parallel lines of the trajectory of reflected light spot on the receiver plane; (2) there is a linear relation between motors' displacements and that of reflected light spots on the receiver plane.

To verify these hypotheses, we carried out the following test: a laser pointer is placed in front of a facet emitting light approximately parallel to $z$-axis; the reflected light by the facet is viewed on a screen (perpendicular to $z$-axis) placed near the focus of the mirror (see Figure 3(a)). Controllable step motors connected to footholds $\left(M_{1}, M_{2}\right)$ enable us to fine-control their displacement. We first moved two motors $24 \mathrm{~mm}$ forward $(+)$ with an interval of $2 \mathrm{~mm}$. After each move, the position of light spot on the screen was marked. We repeated this at several different initial states (initial position of $M_{1}, M_{2}$ ). The trajectories are shown in Figure 3(b) (approximately horizontal). As the rotating axis is much closer to the motors when we move one motor forward and one backward simultaneously, we moved $5 \mathrm{~mm}$ simultaneously $M_{1}$ forward while $M_{2}$ backward with an interval of $1 \mathrm{~mm}$. This gives the same order of displacement 


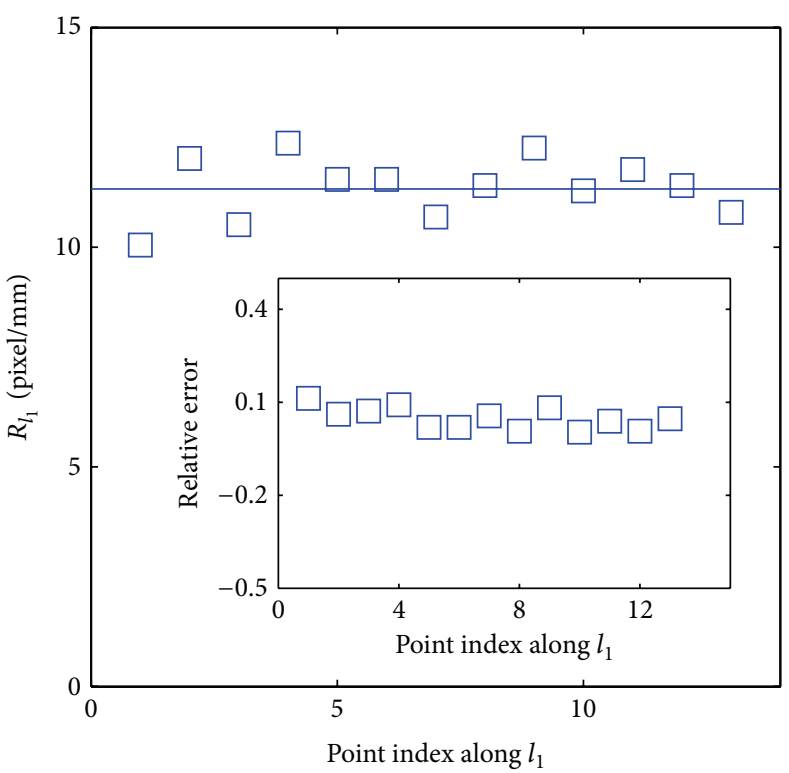

(a)

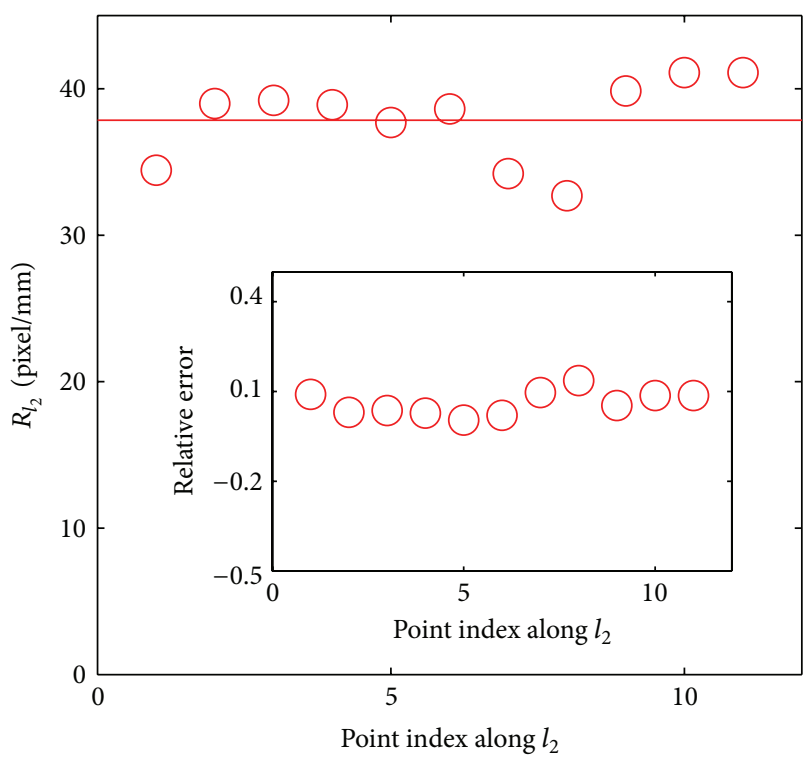

(b)

FIGURE 4: Ratio between the distance of light spots in the image and the motors' displacement along line $l_{1}$ (a) and line $l_{2}$ (b). Solid lines show the average values calculated using only the start and end position of the movements. The insets show the relative error of representing the ratios by their average values.

of light spots on the screen as moving two motors $24 \mathrm{~mm}$ along the same direction and forms a trajectory almost normal to the previous ones (Figure 3(b)).

The spots on the screen were recorded with a digital camera $(480 \times 640$ pixel $)$ for detailed analysis. Using the coordinate (0-640 pixel from left to right, 0-480 pixel from up to down) of the image, the position of each point can then be fully determined. Applying the Least Squares Method [9], we found that the trajectories of the light spots on the screen are well fitted by straight lines, with relative error of $10^{-2}$. As an example, we show in Figure 3(c) that the recorded data are well fitted with a straight line and their relative errors in Figure 3(d). We notice that as long as we move both two positively (or one positive and one negative), the trajectories are indeed parallel to each other.

To get deeper insight on how the spot's trajectory on the screen depends on the motors displacement, in Figure 4 we plot the ratio $\left(R_{l_{1}}, R_{l_{2}}\right)$ between the pixel distance resulting from the movement of the motors and their displacement. One sees clearly that the same amount of motor displacements gives rise to same displacement of light spots on the image: along line $l_{1}$ the average $\left\langle R_{l_{1}}\right\rangle=11.36 \mathrm{pixel} / \mathrm{mm}$ with a standard deviation $\sigma_{l_{1}}=0.69$; along line $l_{2}\left\langle R_{l_{2}}\right\rangle=$ $38.90 \mathrm{pixel} / \mathrm{mm}$ with a standard deviation $\sigma_{l_{2}}=2.85$. These two values can be approximated by the mean ratio defined as the distance from the initial point to the terminal point divided by the motor displacement (solid line), with a relative error around $10 \%$ along both $l_{1}$ and $l_{2}$ (see insets of Figure 4 ). We also noticed that the ratio between displacement of light spot on the screen and that of the required motors' displacements changes little, with a mean value of $1.72 \mathrm{~cm} / \mathrm{mm}$ and a relative error of $\sim 10 \%$.

\section{Test}

A test of the proposed strategy is carried out in laboratory, on a dish concentrator of a $25 \mathrm{~kW}$ Stirling Energy System with a surface area of about $90 \mathrm{~m}^{2}$. The entire dish consists of 40 facets, lying on top of 15 arms, with 13 facets inside and 27 outside. In the test, we only used 3 arms, which can only support 4 facets, with one inside and three outside.

During the test, a laser point $(0.5 \mathrm{~mW}, \phi=5 \mathrm{~mm})$ is projected onto the near the geographical center of facet. A receiver plane is placed about $l_{s}=50 \mathrm{~cm}$ away from the focus point. The reflected light spot on the plane is then captured by a camera (Jinghang $480 \times 640$ industrial USB camera) and sent to a computer (Figure 5). As the size of the reflected light spots on the receiver plane is related to the quality of facet, we compute the position of the light spot $R\left(x_{R}, y_{R}\right)$ as the mass center of the illumination of the photo (panel (b)) [10]. We then implement the displacements given by (3) under Labview (Version 12.0) environment. Combining a fine-selected step motor, a precision as small as $0.006 \mathrm{~mm}$ can be achieved, which gives an alignment error less than $1 \mathrm{mrad}$ $(\arctan ((0.006 \times 1.72) / 50))$.

To guarantee an even distribution of flux energy, the focus of each facet should lie within a specific region (target) around the center of the aperture of the receiver. The purpose of alignment is to adjust light spot to a given position quickly and accurately. To make our test more general, targets on a screen $(60 \mathrm{~cm} \times 60 \mathrm{~cm})$ located near the focus are randomly selected.

3.1. Alignment Procedures. As mentioned in Section 2, to the determine the movements of each motor, some prerequisites 


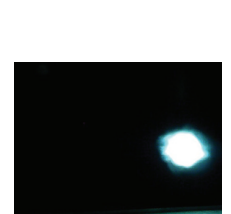

(b)

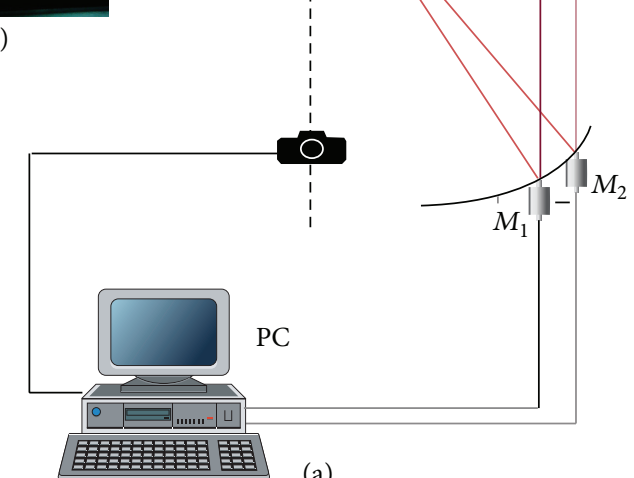

(a)

FIGURE 5: Schematic illustration of experiment setup. Light from a remote source is projected to a facet. The spot of the reflected light is captured by a camera (panel (b)) and sent to computer to compute its center $R$. Using $R$ and target position $T$, the displacements that eliminate the deviations between $R$ and $T$ are sent to motors $M_{1}$ and $M_{2}$.

are needed. These include the ratios $R_{l_{1}}, R_{l_{2}}$, slopes $k_{1}, k_{2}$, and direction vectors $\hat{i}, \hat{j}$.

To determine these quantities, for any facet we introduce a procedure called "initialization": withdraw the two footholds $M_{1}$ and $M_{2}$ to the innermost position and record the position of light spot $A\left(x_{a}, y_{a}\right)$; then simultaneously move $M_{1}$ and $M_{2}$ forward by a distance $d_{1}$ which leads the spot to move to position $B\left(x_{b}, y_{b}\right)$. After having recorded the spot at $B$, we then move $M_{1}$ forward and $M_{2}$ backward by distance $d_{2}$. This is accompanied by a change of light spot from $B$ to position $C\left(x_{c}, y_{c}\right)$ (Figure 2). The prerequisites $R_{l_{1}}, R_{l_{2}}$, and $\hat{j}, \hat{i}$ are then calculated as

$$
\begin{aligned}
& R_{l_{1}}=\frac{\sqrt{\left(x_{a}-x_{b}\right)^{2}+\left(y_{a}-y_{b}\right)^{2}}}{d_{1}}, \\
& R_{l_{2}}=\frac{\sqrt{\left(x_{b}-x_{c}\right)^{2}+\left(y_{b}-y_{c}\right)^{2}}}{d_{2}}, \\
& \hat{j}=\frac{\left(x_{a}-x_{b}\right) \hat{i}_{x}+\left(y_{a}-y_{b}\right) \hat{i}_{y}}{\sqrt{\left(x_{a}-x_{b}\right)^{2}+\left(y_{a}-y_{b}\right)^{2}}} \\
& \hat{i}=\frac{\left(x_{b}-x_{c}\right) \hat{i}_{x}+\left(y_{b}-y_{c}\right) \hat{i}_{y}}{\sqrt{\left(x_{b}-x_{c}\right)^{2}+\left(y_{b}-y_{c}\right)^{2}}}
\end{aligned}
$$

and the slopes $k_{1}=\left(y_{b}-y_{a}\right) /\left(x_{a}-x_{b}\right), k_{2}=\left(y_{c}-y_{b}\right) /\left(x_{b}-x_{c}\right)$. Here $\hat{i}_{x}$ and $\hat{i}_{y}$ are direction vectors along $x$-axis and $y$-axis, respectively.

With these quantities known, the alignment can be carried out. We first calculate the deviation between the current light spot $R$ to the target $T, d_{R T}$. If it is larger than a critical

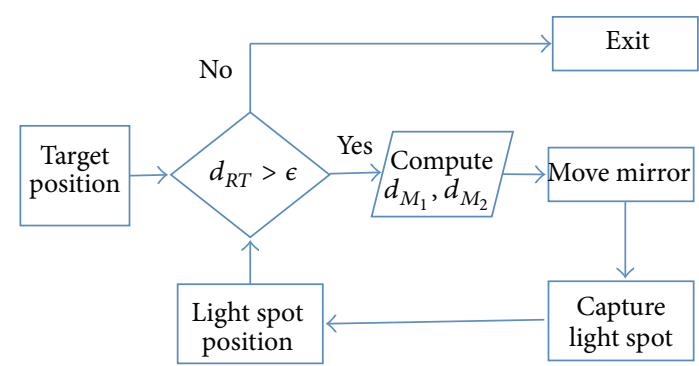

Figure 6: Control architecture of the dish alignment method.

value $\epsilon$, adjustments are carried out by moving the footholds $M_{1}$ and $M_{2}$ with distance given by (3). This procedure is repeated until $d_{R T}<\epsilon$ (a closed-loop system; see Figure 6 for more detailed control architecture).

With constraint by precision of motor controller, the final error is determined by the light spot displacement $l_{p}$ on the receiver plane resulting from moving motor one precise step $\left(l_{p_{\text {min }}}=\right.$ motor controller precision $\times R_{l_{x}}, x$ being 1 or 2$)$. Thus, to achieve a desired alignment precision error $\sim \mathrm{mrad}$ [7], the critical value $\epsilon$ in pixel is given as

$$
\epsilon=\frac{\text { error } * l_{s}}{l_{p_{\min }}} .
$$

3.2. Results. A test was first carried out on a facet on the out layer. Within the image coordinate, the initialization procedure (moving both $20 \mathrm{~mm}$ forward and $5 \mathrm{~mm}$ in opposite direction) gives $R_{l_{1}}=4.387, R_{l_{2}}=24.821, k_{1}=-2.795, k_{2}=$ 0.1841 , and $\hat{j}=0.3316 \hat{i}_{x}-0.9434 \hat{i}_{y}, \hat{i}=0.9834 \hat{i}_{x}+0.1815 \hat{i}_{y}$.

With a randomly selected target $T$ (purple $\square$ ), Figure 7(a) shows how the light spot (black $\bullet$ ) approaches the ideal target location. One sees that after each adjustment, the distance to the target $d_{R T}$ decreases exponentially. This can be explained by the systematic errors resulting from the representation of $R_{l_{1}}$ (and $R_{l_{2}}$ ) using three light spots $A, B$, and $C$. These results give us the confidence that our method is robust and can be used to adjust all facets to their desired locations.

To eliminate these errors, and eventually get a quicker convergence to the target, we elongated the motor displacements during initialization. In this case, the light spot (initially at $R(274.9,230.2))$ reaches a target at $T(375,213)$ immediately. We then applied this approach to other facets and other target locations. It was found that the alignment stops after about 4 loops of adjustment.

To apply this method to a field alignment and guarantee its accuracy, there are several issues that need to be addressed. First, light projected onto each facet should be parallel to the dish axis. Second, points used to do the alignment should represent the physical property of the facet. To solve these problems, we first adjusted the dish axis to be exactly vertical by hanging a hammer at the center of receiver plane and tuning the bolts of the rotating structure which holds the whole dish. We then put large diameter a set of laser beams $(\Phi=50 \mathrm{~mm}, \lambda=632 \mathrm{~nm}$, and divergence angle less than $0.01 \mathrm{mrad}$ ) on a separate holding structure and adjusted all 


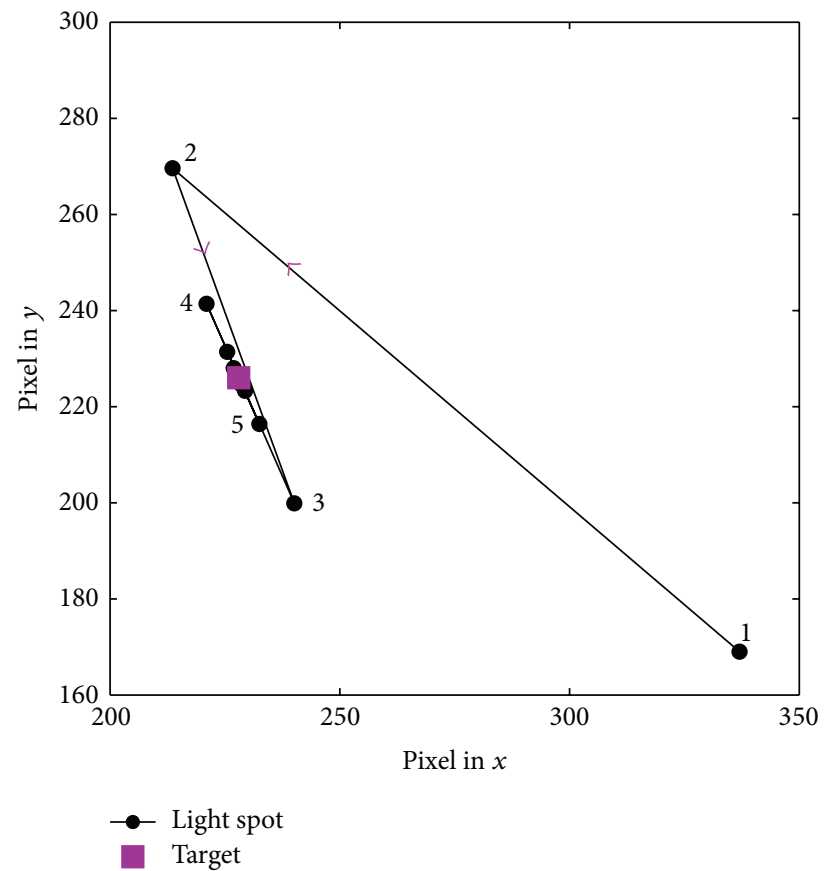

(a)

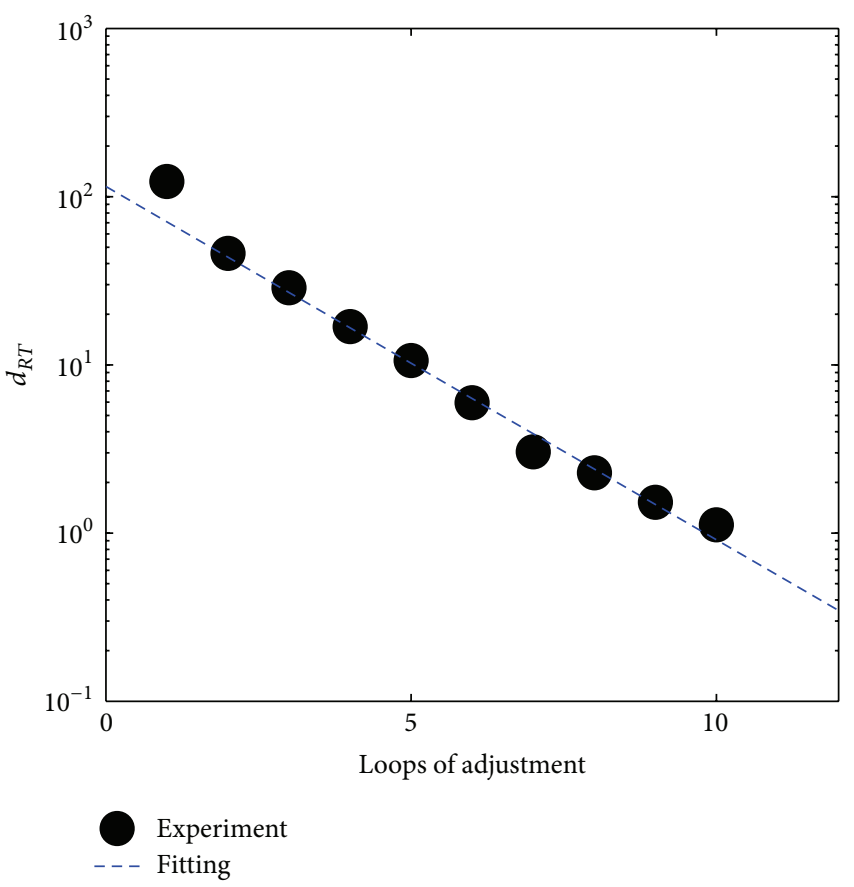

(b)

FIGURE 7: (a) The position of light spots $\left(\bullet\right.$ after each adjustment and the target (purple $\square$ ) on the screen. (b) Distance to the target $d_{R T}(\bullet)$ after each adjustment shows an exponential decline (dash line).

laser beams to be vertical using a laser plumb instrument. With these settings, we guarantee that each facet is aligned with light parallel to dish axis by simply rotating the dish using the existing azimuth driver.

\section{Conclusion and Discussion}

A new method has been proposed for the alignment of faceted solar dish concentrator. This method is based on a mechanical structure of the footholds of the facet, which forms an isosceles triangle configuration. Moving the two bottom-footholds in same or opposite directions, light spot on a screen near the focus of the facets forms linear trajectories. Fixed ratios between light spot displacements and foothold movements allow a fine control of step motors and eventually an automatic adjustment.

Moving reflected light spots of each facet to their desired locations is not only an issue of efficiency of energy conversion but also a requirement of long-time safe operation, as any overlap of facets would potentially damage the gas tube of the heat-head (heat-absorbing plane of the PCU). The method proposed in this paper addresses the issue of how to move the reflected light spots to their desired locations, which can be predesigned using computer software [11]. Accurately moving the spots to match the target points leads to a good energy usage [12].

Our method shows significant advantages comparing to other alignment approaches as mentioned in Section 1. This approach allows a quick (less than 4 loops) and accurate alignment to any part of the concentration. As the motor takes less than one minute to move the foothold from innermost to outermost $(\sim 4 \mathrm{~cm})$, a complete alignment procedure finishes within 5 minutes with an accuracy of $1 \mathrm{~mm}$ (one pixel) under our experimental configuration. Furthermore, as the dish is put vertically, operators can easily attach motors to facets without climbing onto the holding structure with a lift which may be as high as $12 \mathrm{~m}$. With the fixed vertical laser sources, no movements have to be made for all the equipment. After one facet has been done, simply rotating the dish switches to the next one. The technique frees the workload of an observer by using a camera and a computerbased image processing for extracting spot center. It reduces the ambiguity from individual to individual. Furthermore, as mass center of the converged light spot is used to determine the current position of a facet, effect of slope error (local defection of facet surface quality) can be averaged out by using large area of illumination; that is, put multiple laser points above each facet.

Although our method is based on the isosceles triangle structure of footholds that support old generation facet, it is also applicable to the recent industrial installations with four footholds facet: adjusting three of them rather than two, which would result in rotations of facet with two perpendicular axes. This scheme might be used to helistat facets [13], as their two-axis tracking structure provides natural rotating axis.

There also several limitations. First, poor accuracy during initialization leads to more loops to get close enough to the desired location. To obtain accurate values of the prerequisites, motors are supposed to move as long as possible, 
as the values of $R_{l_{1}}, R_{l_{2}}$ and $k_{1}, k_{2}$ fluctuate from region to region (see Figures 3 and 4 ). This put requirements on installation: footholds should be at the innermost position. In practice, this usually requires in addition adjustments of the third foothold. Second, the method is vulnerable to wind. Under windy conditions, the light spot may fluctuate around the target. Manual termination is then required. Third, to guarantee each facet have same parallel light, the pedestal supporting the dish needs to be vertical. In practice, we use a hammer hanging from the centre of the receiver plane. We then adjust the bolts on the holding structure to ensure that the trajectory of the tip of the hammer formed by rotating the dish forms a circle with a diameter less than $50 \mathrm{~mm}$, which contributes to an estimated error in the order of mrad. This procedure is time-consuming.

\section{Conflict of Interests}

The authors declare that there is no conflict of interests regarding the publication of this paper.

\section{Acknowledgments}

The authors thank Hangzhou Solarversal company for providing experiment facilities. This work is partially supported by Zhejiang Provincial Natural Science Foundation under Grant LQ14A050002 and NSFC under Grant 61374056.

\section{References}

[1] S. K. Kim, J. H. Jeon, C. H. Cho, E. S. Kim, and J. B. Ahn, "Modeling and simulation of a grid-connected PV generation system for electromagnetic transient analysis," Solar Energy, vol. 83, no. 5, pp. 664-678, 2009.

[2] T. M. Razykov, C. S. Ferekides, D. Morel, E. Stefanakos, H. S. Ullal, and H. M. Upadhyaya, "Solar photovoltaic electricity: current status and future prospects," Solar Energy, vol. 85, no. 8, pp. 1580-1608, 2011.

[3] P. Würfel and U. Würfel, Physics of Solar Cells: From Basic Principles to Advanced Concepts, John Wiley \& Sons, New York, NY, USA, 2009.

[4] "Solar power stirling engine," http://www.ejsong.com/mdme/ memmods/MEM23041A/thermo/heat_engines_files/Solar_Stirling.html.

[5] C. E. Andraka, J. Yellowhair, and B. D. Iverson, "A parametric study of the impact of various error contributions on the flux distribution of a solar dish concentrator," in Proceedings of the 4th International Conference on Energy Sustainability (ES '10), pp. 565-580, ASME, May 2010.

[6] J. B. Blackmon and K. W. Stone, "Application of the digital image radiometer to optical measurement and alignment of space and terrestrial solar power systems," in Proceedings of the 28th Intersociety Energy Conversion Engineering Conference, pp. 563-570, Atlanta, Ga, USA, August 1993.

[7] C. E. Andraka, R. B. Diver, and K. S. Rawlinson, "Improved alignment technique for dish concentrators," in Proceedings of the International Solar Energy Conference (ISEC '03), Hawaii Island, Hawaii, USA, 2003, SAND2003-0258C.

[8] C. E. Andraka, J. Yellowhair, K. Trapeznikov et al., "AIMFAST: an alignment tool based on fringe reflection methods applied to dish concentrators," Journal of Solar Energy Engineering, vol. 133, no. 3, Article ID 031018, 6 pages, 2011.

[9] D. Borowiak, "Linear models, least squares and alternatives," Technometrics, vol. 43, no. 1, p. 99, 2001.

[10] R. A. Serway, J. S. Faughn, and C. Vuille, College Physics, vol. 1, Cengage Learning, 9th edition, 2011.

[11] V. J. Remero, "CIRCE2/DEKGEN2: a software package for facilitated optical analysis of 3-D distributed solar energy concentrators," Sandia National Laboratories Report, SAND, 1984.

[12] J. Yellowhair and C. E. Andraka, "Projection of flat-plane fluxmap images onto non-flat receiver surfaces," in Proceedings of the 5th ASME International Conference on Energy Sustainability, Paper no. ES2011-54153, pp. 485-494, Washington, DC, USA, August 2011.

[13] R. Buck and E. Teufel, "Comparison and optimization of heliostat canting methods," Journal of Solar Energy Engineering, Transactions of the ASME, vol. 131, no. 1, pp. 0110011-0110018, 2009. 


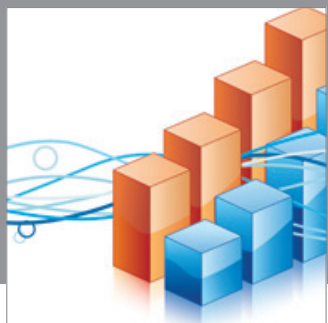

Advances in

Operations Research

mansans

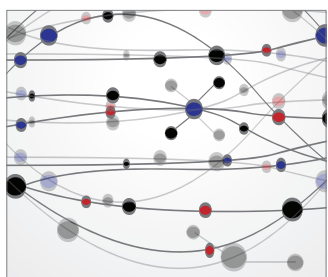

The Scientific World Journal
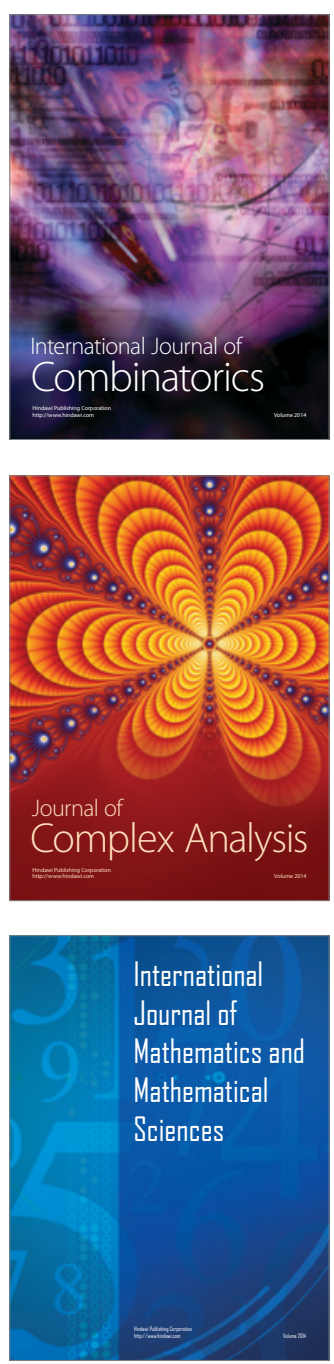
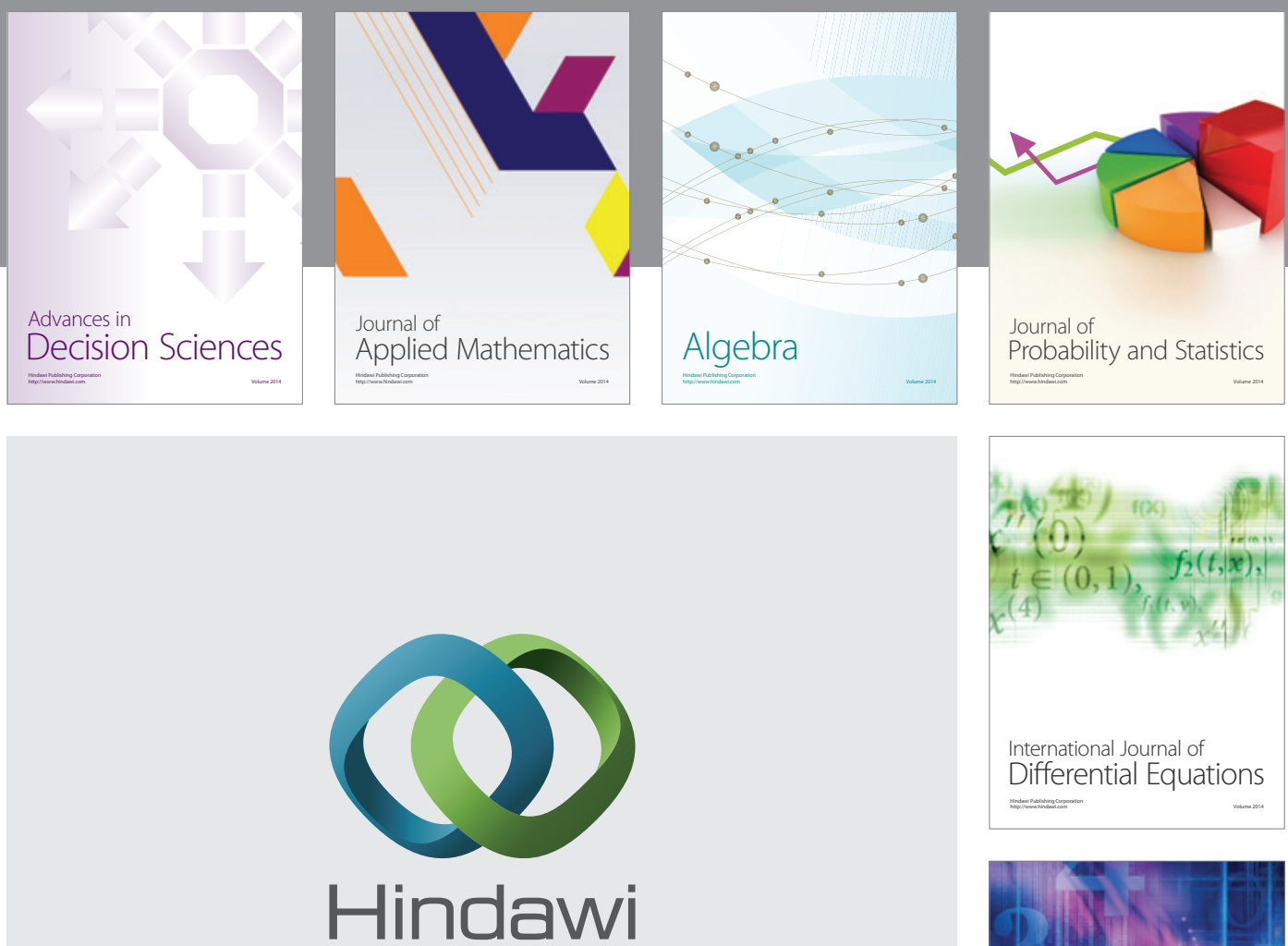

Submit your manuscripts at http://www.hindawi.com
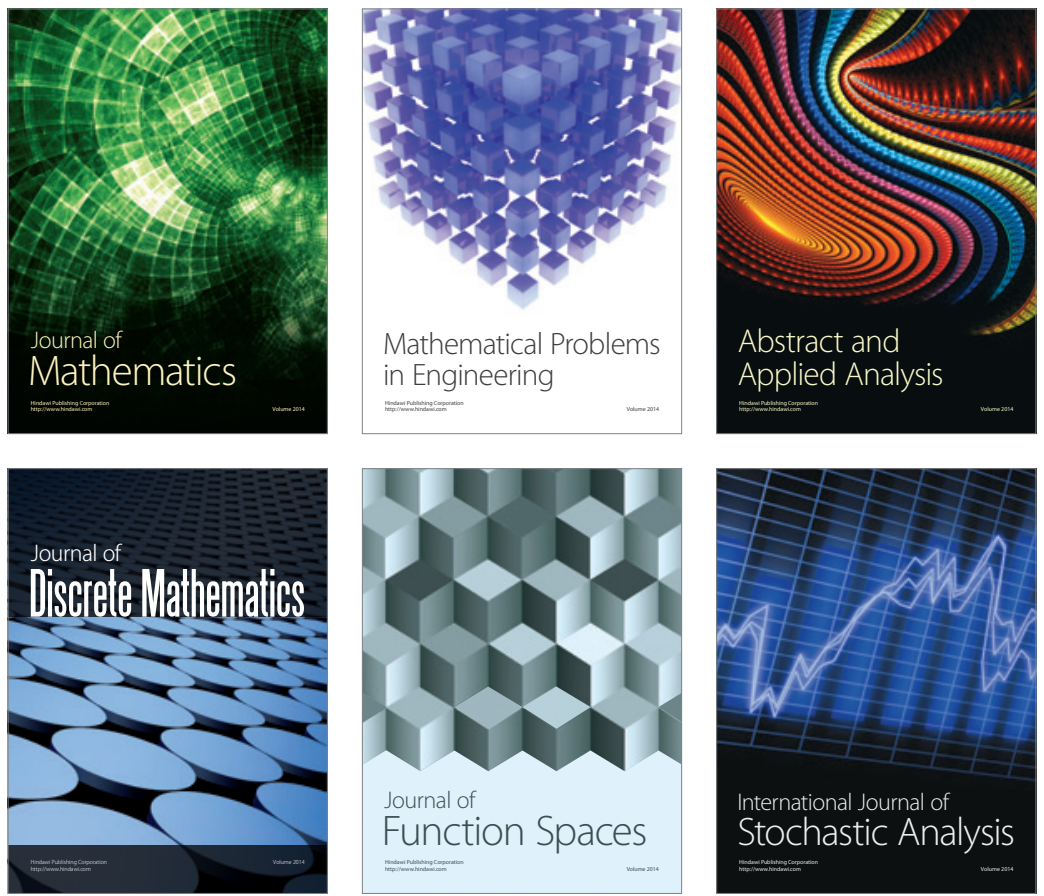

Journal of

Function Spaces

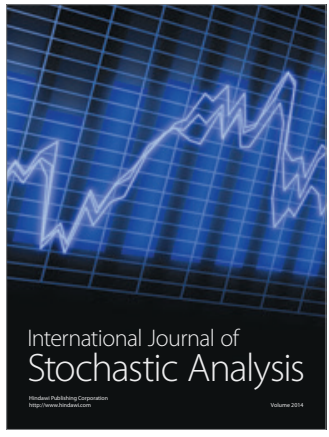

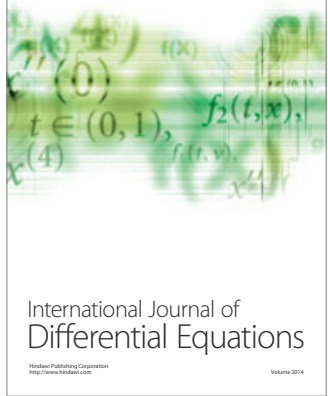
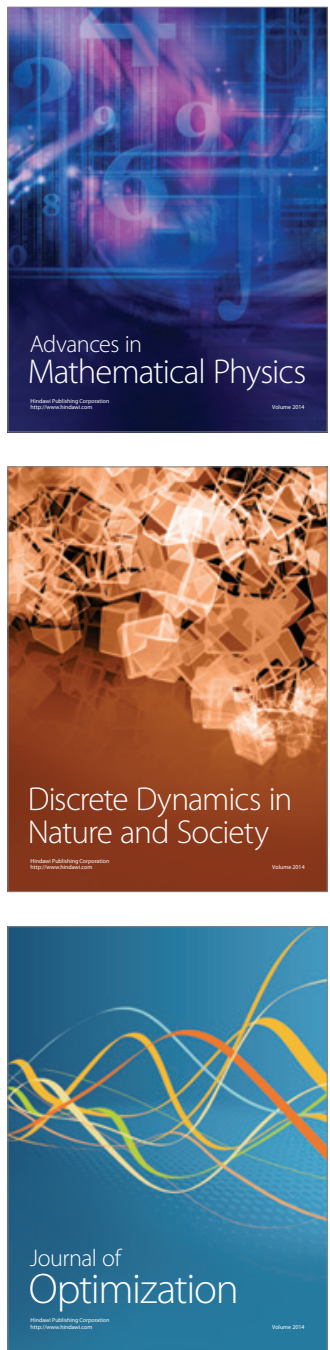